\title{
Building blocks of an artificial kagome spin ice: Photoemission electron microscopy of arrays of ferromagnetic islands
}

\author{
E. Mengotti, ${ }^{1}$ L. J. Heyderman,,${ }^{1} *$ A. Fraile Rodríguez, ${ }^{1}$ A. Bisig, ${ }^{1}$ L. Le Guyader, ${ }^{1}$ F. Nolting, ${ }^{1}$ and H. B. Braun ${ }^{2}$ \\ ${ }^{1}$ Paul Scherrer Institut, CH-5232 Villigen PSI, Switzerland \\ ${ }^{2}$ School of Physics, University College Dublin, Belfield, National University of Ireland, Dublin 4, Ireland
}

(Received 26 June 2008; published 3 October 2008)

\begin{abstract}
Arrays of dipolar coupled ferromagnetic islands arranged in specific geometries provide ideal systems to directly study frustration. We have examined with photoemission electron microscopy the magnetic configurations in three basic building blocks of an artificial kagome spin ice consisting of one, two, and three rings. The kagome spin ice arrangement is particularly interesting because it is highly frustrated and the three interactions at a vertex are equivalent. Employing dipolar energy calculations, we are able to make a full characterization of the magnetic states and therefore identify the lowest energy states. Experimentally we find that the ice rule is always obeyed even at low dipolar coupling strengths. However, as the number of rings increases there is a drastic decrease in the ability to achieve the low-energy states via demagnetization, a behavior also identified in the magnetization reversal. This carries the implication that the ground state will never be achieved in the infinite system. Finally, we show that at low coupling, the applied field direction governs the resulting states. This work opens the door to a novel class of systems for future spintronic applications.
\end{abstract}

DOI: 10.1103/PhysRevB.78.144402

PACS number(s): 75.75.+a, 75.25.+z, 75.60.Jk, 75.70.Ak

\section{INTRODUCTION}

Frustration governs the properties of many different physical systems and, due to its unfathomable character, has captured the fascination of scientists working in several different research fields. This is a universal phenomenon which occurs when not all interactions in a system can be satisfied at the same time, for example underpinning the configuration of microdomains in diblock copolymers, ${ }^{1}$ the phase behavior of liquid crystal structures, ${ }^{2}$ the coexistence of ferroelectricity and magnetism in some multiferroic materials, ${ }^{3}$ and the behavior of bulk magnetic spin systems, a notable example being the pyrochlore spin ice, ${ }^{4}$ so-called because of the similarity to proton ordering in water ice. ${ }^{5}$ For all of these frustrated systems, a great deal of knowledge could be gained if a direct observation of the frustrated configurations were available, which is why recent research has been directed to the study of dipolar coupled ferromagnetic island arrays arranged in two-dimensional frustrated geometries. ${ }^{6-8}$ These are created by state-of-the art lithography techniques and, employing various imaging techniques, the magnetic states can be directly observed. A striking example of an artificially created frustrated system is the spin ice consisting of elongated ferromagnetic islands placed on a square lattice. ${ }^{6}$ The shape and size of the islands is chosen to ensure that they each have a single magnetic domain state and this system was found to exhibit short-range correlations, displaying the characteristics of the bulk spin ice.

In the current work, we focus our attention on another artificially created frustrated geometry: the kagome spin ice, ${ }^{9,10}$ where the elongated ferromagnetic islands are placed on the sites of a kagome lattice, so forming the links of a honeycomb [see Fig. 1(a)]. The kagome system is not only highly frustrated but has the advantage over the squarelattice spin ice that the interactions between the three islands at a vertex are equivalent. ${ }^{9}$ The so-called ice rule dictates that
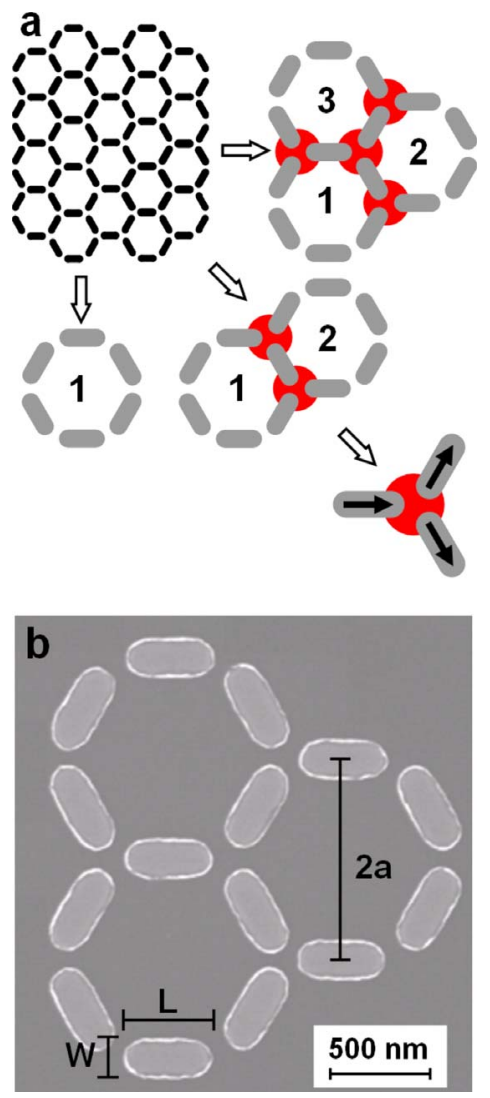

FIG. 1. (Color online) The artificial kagome spin ice building blocks. (a) The three basic building blocks consisting of one, two, and three rings extracted from the infinite array. The three-island vertices are highlighted with red disks and an example of one of the one-in/two-out spin ice moment configurations is given. (b) SEM image of a three-ring kagome structure fabricated from a $20 \mathrm{~nm}$ thick permalloy sample with island type III $(W=200 \mathrm{~nm}$, $L=470 \mathrm{~nm}, a=500 \mathrm{~nm}$ ). 


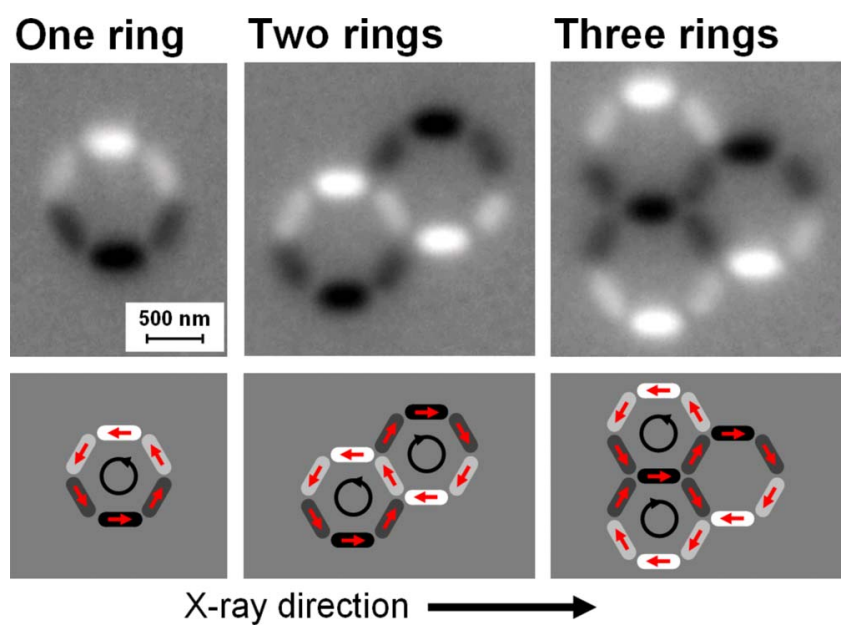

FIG. 2. (Color online) XMCD images of the ground states of the artificial kagome spin ice building blocks. The single contrast associated with each island indicates that they are monodomain with the magnetic moment aligned parallel to the island long axis. The x-ray propagation direction is indicated (horizontal). Islands with moments parallel (antiparallel) to the x-ray direction, i.e., pointing to the right (left), have a black (white) contrast. Islands with moments at $\pm 60^{\circ}$ or $\pm 120^{\circ}$ to the $\mathrm{x}$-ray polarization direction have one of two intermediate contrasts: dark gray or light gray, respectively. The four contrast levels in a single XMCD image allow the unequivocal determination of the magnetic states given schematically below each image.

either two moments point toward and one points away from the vertex (two-in/one-out), or vice-versa (one-in/two-out). This results in six possible configurations ${ }^{10}$ [one of which is shown in Fig. 1(a)] where a full compensation of the local stray fields is not possible. By focusing on the building blocks, ${ }^{11,12}$ extracting the one-, two-, and three-ring structures from the infinite kagome system as shown in Fig. 1(a), we are able to determine the energies of all of the possible states using a dipolar interaction model. Here we represent each island with a single magnetic moment, made possible by the fact that the monodomain islands have a finite size and are isolated, in contrast to a kagome network where domain walls are present. ${ }^{7,8}$ Employing X-ray magnetic circular dichroism (XMCD) in a photoemission electron microscope (PEEM), we are able to directly observe the magnetic configuration in each individual island and to confirm that they are single domain (Fig. 2). Following a demagnetization process involving rotation of the sample in a magnetic field, we determine the frequency of states as a function of the dipolar coupling strength between the islands, which we modify by varying the lattice parameter, $a$ [see island geometry in Fig. 1(b)]. We find the important result that, as the number of rings increases, there is a dramatic reduction in the ability to achieve the low-energy states, which leads us to conclude that it will be extremely difficult to achieve the ground state as the number of rings approaches infinity. Finally we show that this behavior is mirrored by the ability to switch via the low-energy states in an applied magnetic field observed in situ in the PEEM, and that at low coupling strength (high lattice parameter), the field orientation has a high impact on the resulting kagome spin ice configurations.

\section{EXPERIMENTAL METHODS}

In order to fabricate ferromagnetic islands arranged on a kagome lattice [Fig. 1(b)], the pattern was first exposed in an $80 \mathrm{~nm}$-thick layer of polymethylmethacrylate (PMMA) resist spin coated on a silicon (100) substrate using a Leica LION LV1 electron-beam writer. Ferromagnetic cobalt and permalloy $(\mathrm{Ni} 80 \% \mathrm{Fe} 20 \%)$ thin films, with a $1 \mathrm{~nm}$-thick aluminum capping layer to prevent oxidation, were then deposited on the patterned resist by dc magnetron sputtering (base pressure $=3 \times 10^{-6} \mathrm{mbar}$, sputter pressure $=2 \times 10^{-3} \mathrm{mbar}$ ). The sputter rate for permalloy was $62.5 \mathrm{~nm} / \mathrm{min}$ and for cobalt was $53.8 \mathrm{~nm} / \mathrm{min}$. Ultrasound assisted lift-off in acetone was then employed to remove the remaining resist together with the unwanted magnetic material. We studied ring structures with the following three island types $[W=$ width and $L=$ length as shown in Fig. 1(b), and $t=$ thickness]:

Type I: cobalt, $W=160 \mathrm{~nm}, L=470 \mathrm{~nm}, t=10 \mathrm{~nm}$;

Type II: cobalt, $W=200 \mathrm{~nm}, L=470 \mathrm{~nm}, t=10 \mathrm{~nm}$;

Type III: permalloy, $W=200 \mathrm{~nm}, L=470 \mathrm{~nm}, t=20 \mathrm{~nm}$.

Magnetic images were obtained with a PEEM at the SIM beamline $^{13}$ of the Swiss Light Source, Paul Scherrer Institut, employing the XMCD effect at the Fe or Co $L_{3}$-Edge. ${ }^{14}$ The intensity is a measure of the angle between the X-ray circular polarization vector and the magnetic moments. Ferromagnetic domains with magnetic moments parallel or antiparallel to the X-ray polarization vector appear black or white in the XMCD image, and domains with magnetic moments at an intermediate angle have an intermediate gray contrast.

For demagnetization, a magnetic field was applied while the sample rotates about an in-plane axis at a frequency of $\approx 2 \mathrm{~Hz}$. The field was then decreased slowly, at $\approx 5 \mathrm{mT} / \mathrm{sec}$, from a field value of $850 \mathrm{mT}$, well above the saturation field of the kagome structures, down to zero. For each demagnetization run, we measured 100 identical onering, 64 two-ring, and 64 three-ring structures, which were placed on the same substrate with sufficient center-to-center distance between the neighboring structures $(3.5 \mu \mathrm{m}$ for the arrays of one-ring structures and $4.5 \mu \mathrm{m}$ for the two- and three-ring structures) so that the stray field interaction between each structure is negligible. To determine the effect of the demagnetization geometry, demagnetization runs were also performed with an out-of-plane rotation axis. The two geometries for the demagnetization are given in Fig. 7(a).

\section{ENERGY STATES}

First we turn to the energy calculations of the different possible states which set the groundwork for interpreting the experimental observations. The elongated ferromagnetic islands investigated here are single domain (see Fig. 2) and can therefore be approximated by a single macroscopic spin. The dipolar energy between two islands is then given by

$$
E\left(\vec{r}_{1}, \vec{r}_{2}, \vec{m}_{1}, \vec{m}_{2}\right)=\frac{1}{\|\vec{r}\|^{3}}\left[\vec{m}_{1} \cdot \vec{m}_{2}-\frac{3}{\|\vec{r}\|^{2}}\left(\vec{m}_{1} \cdot \vec{r}\right)\left(\vec{m}_{2} \cdot \vec{r}\right)\right],
$$

where $\vec{m}_{1}$ and $\vec{m}_{2}$ are the magnetic moments of two interacting islands and $\vec{r}$ is the distance vector between the centers of the islands. The moment of each island in our experiment is 


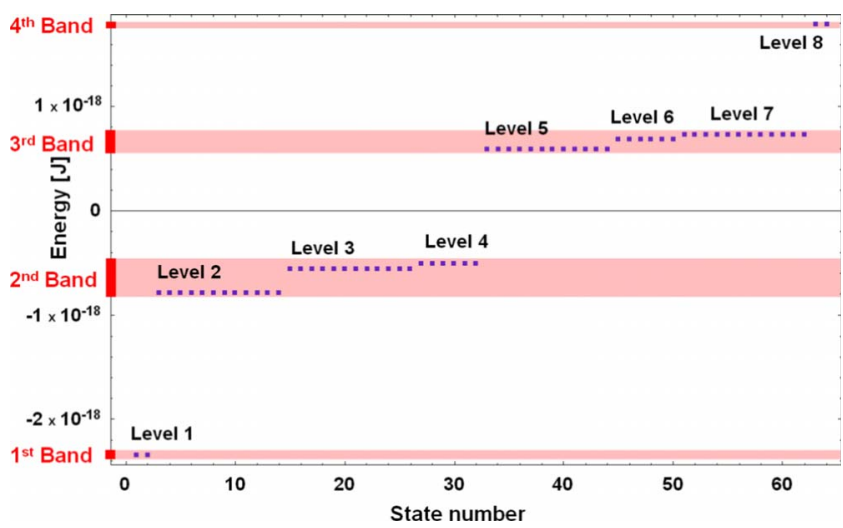

(a)

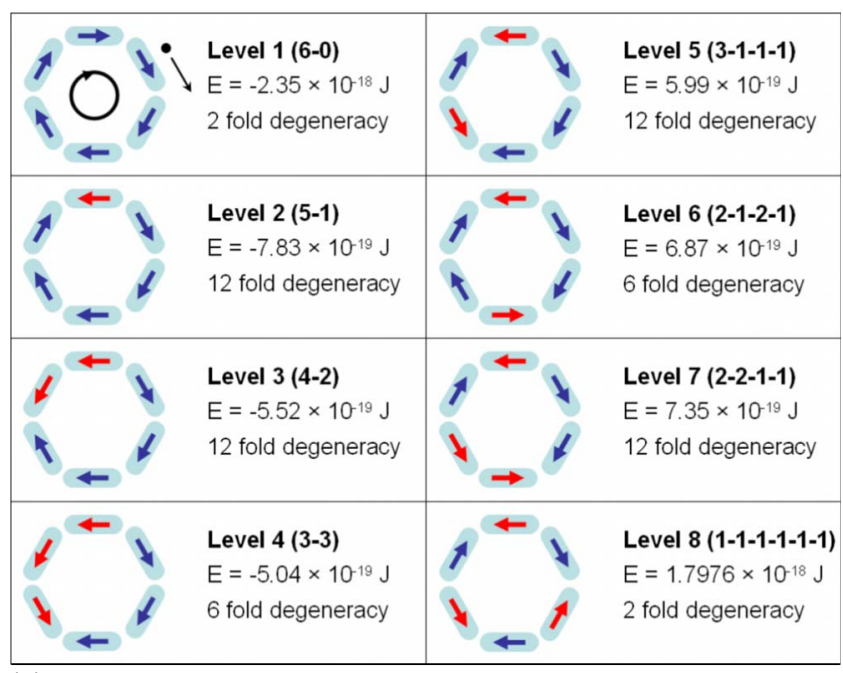

(b)

FIG. 3. (Color online) Energy characterization of the one-ring kagome structure. (a) Calculated dipolar energies of the 64 possible configurations. The energy values refer to $10 \mathrm{~nm}$-thick cobalt islands with $a=500 \mathrm{~nm}, W=200 \mathrm{~nm}, L=470 \mathrm{~nm}$ (island type II). (b) Schematic representation of one degenerate state for each energy level. The states are ordered as a function of increasing energy and labeled $(n-m)$, where $n$ refers to the highest number of neighboring magnetic moments pointing in the same direction around the structure, and $m$ is the number of neighboring magnetic moments pointing in the opposite direction, running clockwise from the starting point indicated with a dot and arrow. For higher states we use the nomenclature $k-l-m-n$ to indicate the number of neighboring moments aligned in the same direction.

of the order of $1 \times 10^{8}$ Bohr magnetons. This gives an interaction energy between nearest neighbors of $10^{-18} \mathrm{~J}$ (equivalent to $7.2 \times 10^{4} \mathrm{~K}$ ) for a lattice parameter $a=500 \mathrm{~nm}$, corresponding to a high coupling strength in the experiment. Taking into account the shape anisotropy, the moment is set in one of two directions parallel to the long axis of the island. For each of the $2^{N}$ possible configurations, where $N$ is the number of islands in the structure, the energy value, which corresponds to the sum of all terms divided by $N$, is calculated and plotted in Figs. 3-5. The states with the same energy are grouped together and ordered with increasing energy from left to right.

For the one-ring kagome structure, six islands are present resulting in $2^{6}=64$ possible states and the energy levels are
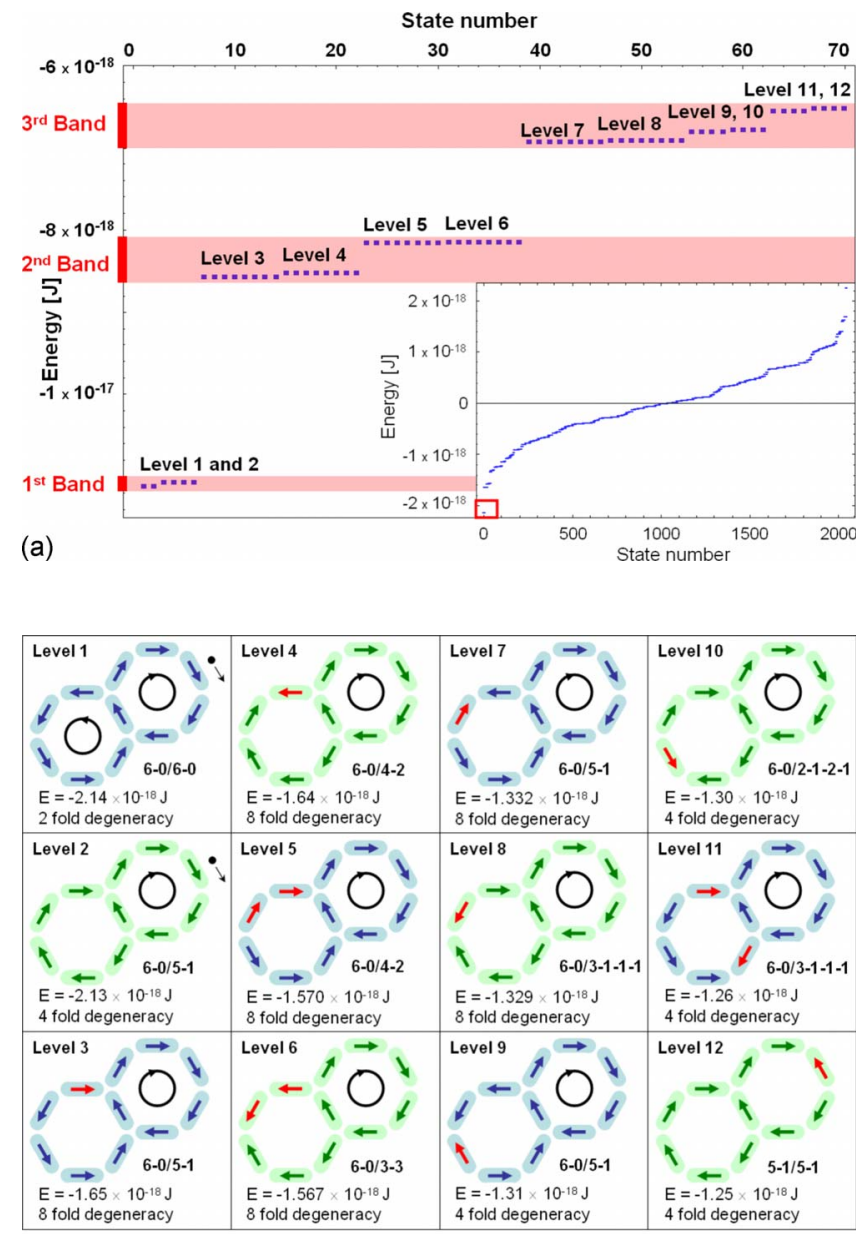

(b)

FIG. 4. (Color online) Energy characterization of the two-ring kagome structure. (a) Calculated dipolar energies of the lowest 70 energy states of the two-ring structure with the same parameters used in Fig. 3 (type II islands, $a=500 \mathrm{~nm}$ ). The energies of all of the 2048 possible states are shown in the inset. (b) Schematic representation of one of the degenerate states for each level up to level 12. In the first energy band, the islands in the double-vortex state (level 1) are colored in blue, and for the external-flux-closure state (level 2), the islands are colored green. The next highest energy states can be derived from these two configurations by simply flipping moments (colored in red) in the individual islands, which are shown in green or blue depending on the source configuration.

grouped in four bands [Fig. 3(a)]. Each pair of neighboring islands is oriented at $120^{\circ}$ to each other and the minimum (maximum) dipolar energy occurs when neighboring moments are aligned head-to-tail (head-to-head or tail-to-tail). Therefore the ground state of a single ring consists of moments circulating either clockwise or anticlockwise. We refer to this energy state as the vortex state due to the similarity with equivalent states in ferromagnetic disks ${ }^{15}$ and rings. ${ }^{16}$ With its twofold degeneracy, the vortex state corresponds to the lowest energy level of the energy plot, labeled level 1, which is simply the first band. Here there is no frustration and the neighbor, the next-nearest-neighbor, and the nextnext-nearest neighbor interactions are all satisfied. We label the levels 1 to 8 in order of increasing energy and we introduce the terminology of the $(n-m)$ state, where $n$ refers to the 


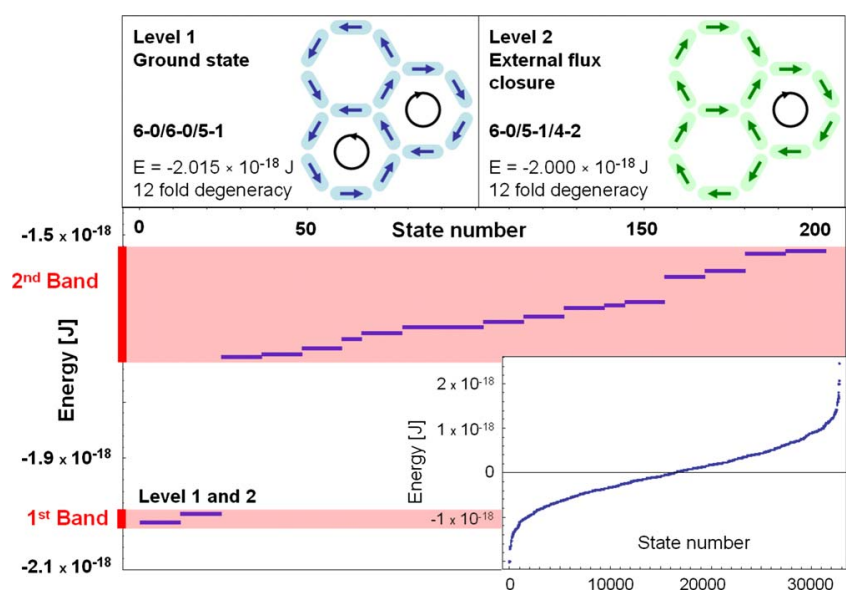

FIG. 5. (Color online) Energy characterization of the three-ring kagome structure. Calculated dipolar energies of the lowest 204 energy states of the three-ring structure with the same parameters used in Fig. 3 (type II islands, $a=500 \mathrm{~nm}$ ). The energies of all of the 32768 possible states are shown in the inset. The lowest two energy states, which belong to the first energy band, are schematically shown at the top of the figure: the islands in the ground state are colored in blue, while the islands in external-flux-closure state are green.

highest number of neighboring magnetic moments pointing in the same direction around the structure, and $m$ is the number of neighboring magnetic moments pointing in the opposite direction. For each energy level, a characteristic configuration, together with the energy value and the degree of degeneracy, is given in Fig. 3(b). For the single ring $n+m=6$, and the vortex state is simply the $6-0$ state. In the next highest band, which is split into three levels $(2,3$ and 4 ), there are 30 possible configurations. The states in this energy band are characterized by having two of the six vertices not in the head-to-tail configuration. Indeed one vertex is in the head-to-head and one is in the tail-to-tail configuration. These levels contain the 5-1, 4-2, and 3-3 states as shown in Fig. 3(b). We refer to the 3-3 state as the "onion state," which is equivalent to the state found in ferromagnetic rings with two domain walls. ${ }^{16}$ The next highest band, including levels 5 to 7 , contains states with two head-to-head vertices and two tail-to-tail vertices. In the highest band (level 8), all vertices have the unfavorable head-to-head or tail-to-tail configuration.

For the two-ring kagome structure, many more states are present $\left(2^{11}=2048\right)$, and frustration is introduced at two vertices with three interacting islands oriented at $120^{\circ}$ to each other [Fig. 1(a)]. Here, $56.25 \%$ of the total number of states (corresponding to 1152 configurations) obeys the ice rule at both vertices. The energies of all of the 2048 possible states, given in detail for the lowest 70 energy states, are shown in Fig. 4(a). The ground state with a twofold degeneracy consists of two vortices (6-0/6-0) with opposite chirality, which we refer to as the double-vortex state. This constitutes level 1 and is shown with the islands colored in blue in Fig. 4(b). The "external-flux-closure" state (6-0/5-1) at level 2 [colored in green in Fig. 4(b)] is very close in energy to the ground state. Here the outer magnetic moments form a chain of moments circulating either clockwise or anticlockwise around the whole two-ring structure, and the central island moment falls into one of the two possible directions resulting in a fourfold degeneracy. Levels 1 and 2 both belong to the lowest energy band shown in Fig. 4(a). From these two basic configurations, the next highest energy states can be derived simply by flipping moments in the individual islands, as indicated by the red arrows in Fig. 4(b).

For the three-ring kagome structure, while two vortices are allowed, it is no longer possible to have three complete vortices. Here only $\sim 32 \%$ of the total $2^{15}=32768$ states (corresponding to 10368 states) have all four three-island vertices [highlighted with red disks in Fig. 1(a)] in a spin ice configuration. The energies of all of the possible configurations, shown in detail for the lowest 204 energy states, are shown in Fig. 5. The lowest energy band consists of two discrete levels. Level 1 corresponds to the ground state which consists of two vortices with opposite chirality and a 5-1 state for the third ring, and level 2 is the external-fluxclosure state with the outer magnetic moments forming a chain of moments circulating either clockwise or anticlockwise around the whole three-ring structure and the three moments in the middle of the structure following the ice rule. In Fig. 5 these states are colored in blue and green, respectively, and both have a 12-fold degeneracy. The next highest energy states contain either two or one vortex.

\section{FREQUENCY OF STATES}

Now we turn to the experimental observations of the moment configurations in arrays of kagome ring structures prepared by electron-beam lithography. The observations are performed employing XMCD in a PEEM. This gives a high magnetic contrast with four distinct gray levels associated with the kagome structure, allowing us to determine unambiguously the direction of the moments in all islands with a single image (Fig. 2). The uniform contrast measured in every island confirms that they are single domain and allows us to use a single dipole approximation to calculate the energy of a given state. We measured the distribution of states in arrays of kagome structures with three different island types (I, II, and III) having different materials and dimensions (see experimental methods section). First, the frequency of observed states versus the coupling strength, $\mathrm{m}^{2} / \mathrm{a}^{3}$, was measured after the samples were demagnetized with rotation of the sample about an in-plane axis as described in the experimental methods section.

For the one-ring kagome structures, practically only the states belonging to the lowest two energy bands [levels 1 to 4 in Fig. 3(a)] were observed following demagnetization and a representative analysis of these states (island type I), including an XMCD image of part of the sample, is given in Fig. 6. For high dipolar coupling strength (low lattice parameter, $a$ ), most of the one-ring structures fall into the vortex ground state, which not only has the lowest energy but also has a zero total magnetization. As the dipolar coupling strength is decreased toward zero, i.e., as $a$ is increased to a high value, we would expect the frequency of a given configuration to reflect the random probability of the moment 

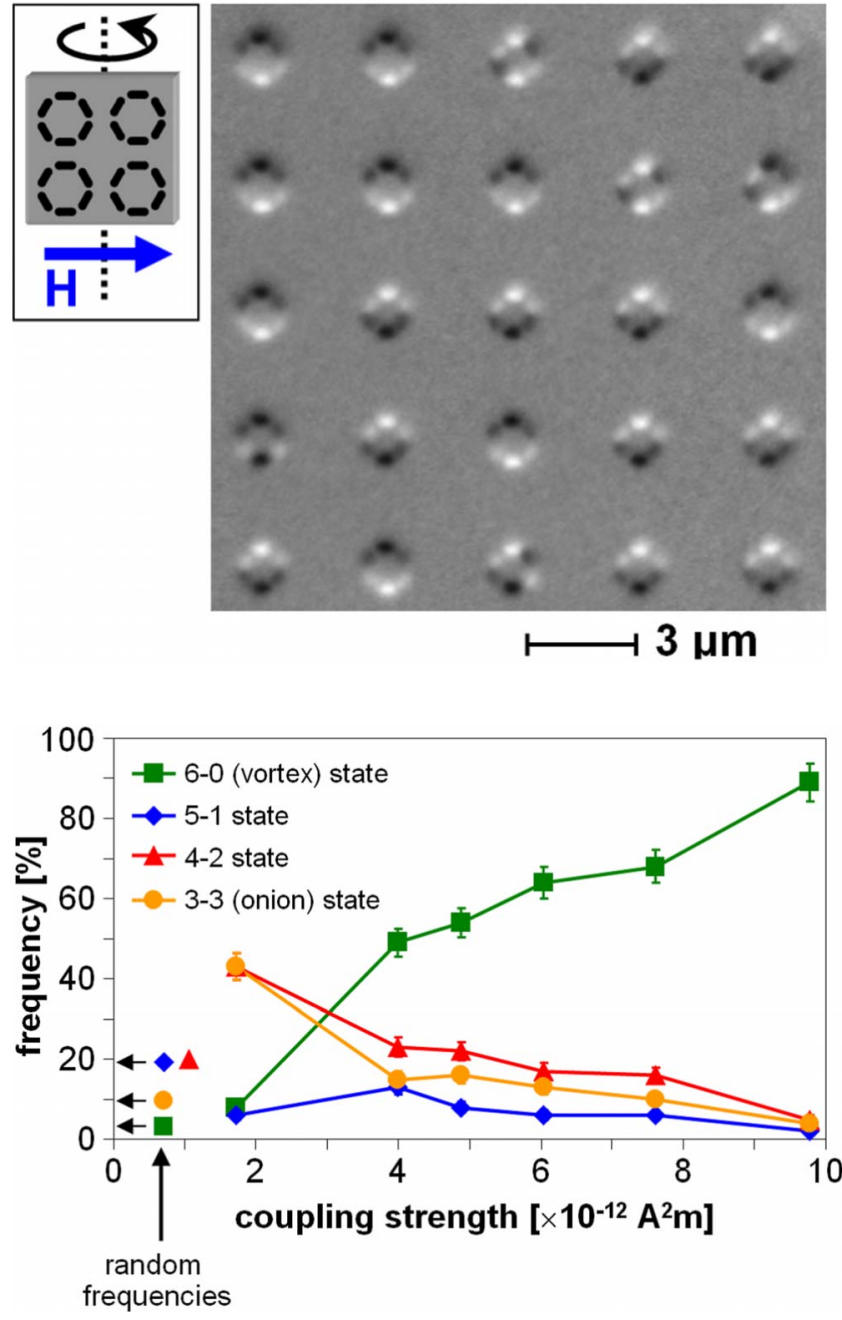

FIG. 6. (Color online) Experimental frequency of states in the one-ring kagome structure following demagnetization. The geometry of the demagnetization setup with the sample rotating about an in-plane axis is shown schematically, and an XMCD image of twenty-five one-ring configurations following demagnetization is given. Below the frequency of observed states is plotted against a measure of the coupling strength, $\mathrm{m}^{2} / \mathrm{a}^{3}$, where $m$ is the moment of each island and $a$ is the lattice parameter. This is a representative result for 100 one-ring kagome structures with island type I, and the standard deviation of each measurement is represented by the error bars. The random frequencies for the different configurations expected at zero coupling, given by the degree of degeneracy divided by the total number of states, are indicated with arrows to the left.

configurations. This is simply given by the degree of degeneracy of each state divided by the total number of states, and indicated in Fig. 6 with arrows. We do indeed see a tendency of the number of ground and 5-1 states to approach the random frequency as the coupling decreases. However, the 3-3 and 4-2 states increase to a value significantly higher than the random expectation. Here the applied field direction governs the behavior at low coupling strengths, favoring states with the moments oriented towards the direction of the applied field (where the majority of islands appear bright or dark in the XMCD image depending on the last field direction before the moments freeze in). As a result, we only
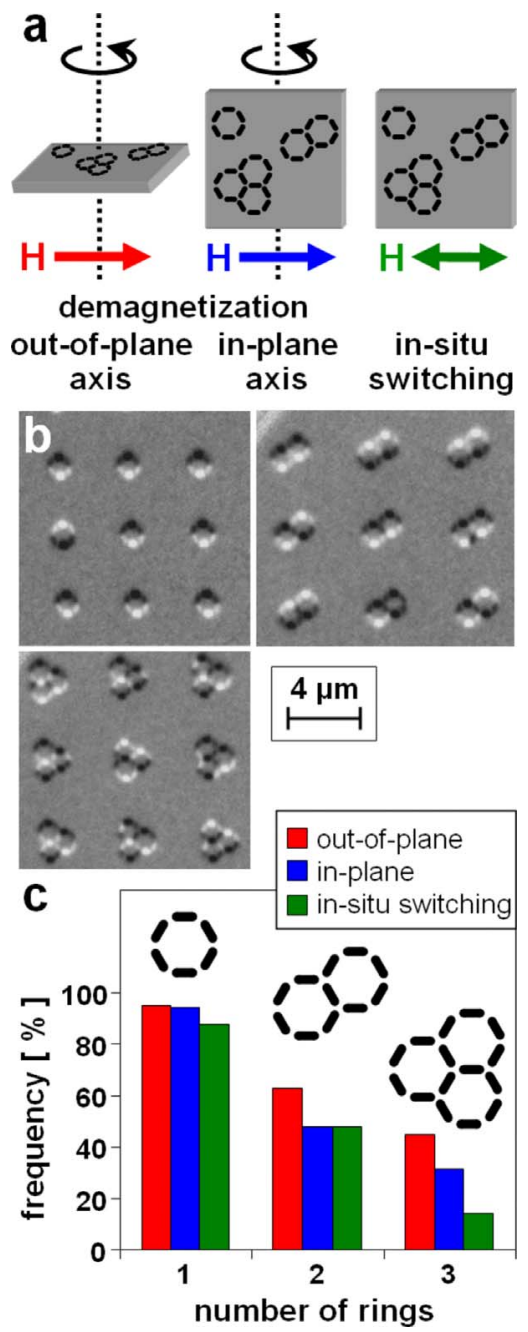

FIG. 7. (Color online) Experimental frequency of low-energy states (band 1) for strongly coupled islands with increasing number of rings. (a) Geometries for demagnetization with in-plane and outof-plane rotation axes and for in situ switching in the PEEM, (b) XMCD images of nine of the one-, two-, and three-ring configurations following demagnetization about an in-plane rotation axis, and (c) the frequency of observed states plotted against the number of rings. These are three representative results of experimental runs where the efficiency (number of single rings in the vortex ground state) was high. The sample and demagnetization geometries for the three runs are as follows: island type I with $\mathrm{m}^{2} / \mathrm{a}^{3}=9.8$ $\times 10^{-12} \mathrm{~A}^{2} \mathrm{~m}$ following demagnetization about an out-of-plane rotation axis (red), island type II with $\mathrm{m}^{2} / \mathrm{a}^{3}=1.1 \times 10^{-11} \mathrm{~A}^{2} \mathrm{~m}$ following demagnetization about an in-plane rotation axis (blue) and island type III with $\mathrm{m}^{2} / \mathrm{a}^{3}=1.2 \times 10^{-11} \mathrm{~A}^{2} \mathrm{~m}$ during switching in situ in the PEEM (green).

observe two of the six possible onion states and four of the twelve 4-2 states.

For the two- and three-ring structures, because of the vast number of available configurations, we do not present a full analysis of all the states present. Here it is more informative to compare the frequencies of low-energy states at high coupling strengths for the different numbers of rings. We measured a sample with kagome structures with type II islands and a high coupling strength corresponding to $\mathrm{m}^{2} / \mathrm{a}^{3}=1.1$ 
$\times 10^{-11} \mathrm{~A}^{2} \mathrm{~m}$, following demagnetization with rotation of the sample about an in-plane axis [see Figs. 7(a)-7(c)]. For the one-ring kagome structures, $94 \pm 2 \%$ fall into the vortex state corresponding to the lowest energy band. For the tworing structures, the percentage of states in the first energy band (including the double-vortex and external-flux-closure states) is $48 \pm 8 \%$. For the three-ring structure the percentage of states in the lowest energy band (ground state with two vortices and external-flux-closure state) is $31 \pm 6 \%$. Since the ability to achieve the low-energy states with our demagnetization method reduces significantly as a function of the number of rings, it is likely that as the number of rings is increased to an infinite value the ground state will never be achieved using this demagnetization method. At low coupling $(a=820 \mathrm{~nm})$, the percentage of the low-energy states for the two and three rings was negligible $(<0.6 \%)$, thus tending to the random values, but again with a directionality of the magnetic configurations (preponderance of bright or dark XMCD contrast) given by the applied field direction when the moments freeze in. The kagome spin ice rule at the three-island vertices (two-in/one-out or vice-versa) is practically always preserved, notably even at low coupling strengths, with the number of three-island vertices which do not follow the ice rule on average for both two and three rings less than $0.2 \%$ (i.e., 1 out of the 584 vertices observed). We have determined the frequency of states for demagnetized samples with all of the different island types, and found that the general behavior was the same. In addition, we demagnetized the same sample four times (with island types I and II) to verify that the distribution of states did not significantly change, and found that each structure did not necessarily fall into the same magnetic state during each demagnetization run.

During the demagnetization process the moments in the ring structures essentially switch back and forth, i.e., between two opposite onion states, when the applied field is above the switching field. In order to observe if this switching occurs via states in the lowest energy band, we directly examined the magnetization reversal in an applied field in situ in the PEEM. The orientation of the applied field is equivalent to that used in the demagnetization with an inplane sample rotation axis, and a schematic of the applied magnetic field geometry for in situ switching is given in Fig. 7(a). Following saturation of the magnetization, we recorded XMCD snapshots of the switching process on applying a reverse field, increasing its value from zero in steps of approximately $6 \mathrm{Oe}$, and recording images at remanence after reducing the field between each step back to zero. For this, we chose islands with a lower coercivity (island type III with $a=540 \mathrm{~nm}$ ) ensuring that they switch in the PEEM magnetizing holder. We followed a full hysteresis loop, applying the field in both directions, and performed measurements on 50 one-ring structures, 32 two-, and 32 three-ring structures. We observed that $88 \%$ of the one-ring structures switch via the vortex state, with $26 \%$ of the one-ring structures switching directly via the vortex state without passing through any other state. For the two-ring structure, the percentage that switch via an energy state in the lowest energy band (doublevortex or external-flux-closure) corresponds to $48 \%$, although the simple path double-onion state to low-energy state to reverse-double-onion state is never followed and always includes a few other states. For the three-ring structure the observed frequency passing through a state belonging to the lowest energy band, i.e., the ground or external-fluxclosure state, was $14 \%$. Notably, the decrease in frequency of observed low-energy states for increasing number of rings in the kagome building blocks is comparable to the decrease in frequency of low-energy states observed following demagnetization with an in-plane sample rotation axis [see Fig. 7(c)]. This implies that the magnetization reversal forms the basis of the demagnetization process. The oscillation of the applied field with decreasing amplitude during the demagnetization process simply allows us to collect all of the lowenergy states, which occur at different field values during switching.

Finally we address the question of whether the geometry of the demagnetization procedure will affect the frequency of observed states, specifically if rotation about an out-of-plane axis in the applied magnetic field will increase the ability to achieve the ground state. It turns out that the results do not significantly differ from those found with an in-plane rotation axis, namely, on increasing the number of rings there is a significant decrease in the number of low-energy states [see Fig. 7(c)]. At low coupling, again a directionality in the configurations is introduced given by the applied field direction before the moments freeze in, with a high proportion of onion and 4-2 states in the single ring structure. For example, in one run with type II islands and a coupling of 2.6 $\times 10^{-12} \mathrm{~A}^{2} \mathrm{~m}$, more than $60 \%$ of the one-ring structures were in the onion or 4-2 state, which is significantly higher than the random expectation value of $28 \%$ and, as before, we only observe two of the six possible onion states and four of the twelve 4-2 states.

\section{CONCLUSION}

By focusing on the building blocks of an artificial kagome spin ice, we have been able to fully characterize the possible energy states employing dipolar calculations and, in particular, we were able to identify the lowest energy states. The single ring is not frustrated and following demagnetization, a high percentage fall into the vortex state demonstrating the high efficiency of the demagnetization procedure. Adding rings introduces frustration at the ensuing three-island vertices where the ice rule is preserved, even at low coupling strengths. We find that as the number of rings increases there is a dramatic decrease in the ability to achieve the lowenergy states, a behavior also identified in the magnetization reversal. Here the system gets trapped in one of the large number of available states and at low interisland dipolar coupling strengths, the orientation of the applied field strongly influences the resulting configurations. ${ }^{10}$ Extrapolating these results, we draw the important conclusion that achieving the ground state will be practically impossible in an infinite system using a demagnetization method involving rotation of 
the sample in a magnetic field. Indeed, in contrast to the spin ice system where the ground state is made up of a series of local vortices, ${ }^{17}$ a maximum of two neighboring vortices is possible, so that only larger flux closure loops will emerge. The knowledge we have gained about the behavior of the kagome spin ice building blocks paves the way for future fascinating studies of these highly frustrated artificial systems and, armed with the complete knowledge of the energy states, we open the door for use of these finite multistate kagome structures in future spintronic devices, either for memory applications or to perform logic operations. ${ }^{18}$

\section{ACKNOWLEDGMENTS}

The authors would like to thank at the Paul Scherrer Institut: Michael Horisberger, Eugen Deckardt, Anja Weber, Christian David, Andrea Steger, and Thomas Neiger for their support, and also at the ETH Zürich: Alessandro Vindigni, Niculin Saratz, and Danilo Pescia for fruitful discussions. This work was supported by the Swiss National Science Foundation and the Science Foundation of Ireland, and part of this work was performed at the Swiss Light Source, Paul Scherrer Institut, Villigen, Switzerland. *laura.heyderman@psi.ch

${ }^{1}$ G. J. Kellogg, D. G. Walton, A. M. Mayes, P. Lambooy, T. P. Russell, P. D. Gallagher, and S. K. Satija, Phys. Rev. Lett. 76, 2503 (1996).

${ }^{2}$ T. Matsumoto, A. Fukuda, M. Johno, Y. Motoyama, T. Yui, S. S. Seomun, and M. Yamashita, J. Mater. Chem. 9, 2051 (1999).

${ }^{3}$ S. W. Cheong and M. Mostovoy, Nature Mater. 6, 13 (2007).

${ }^{4}$ M. J. Harris, S. T. Bramwell, D. F. McMorrow, T. Zeiske, and K. W. Godfrey, Phys. Rev. Lett. 79, 2554 (1997).

${ }^{5}$ L. Pauling, J. Am. Chem. Soc. 57, 2680 (1935).

${ }^{6}$ R. F. Wang, C. Nisoli, R. S. Freitas, J. Li, W. McConville, B. J. Cooley, M. S. Lund, N. Samarth, C. Leighton, V. H. Crespi, and P. Schiffer, Nature (London) 439, 303 (2006).

${ }^{7}$ Y. Qi, T. Brintlinger, and J. Cumings, Phys. Rev. B 77, 094418 (2008).

${ }^{8}$ M. Tanaka, E. Saitoh, H. Miyajima, T. Yamaoka, and Y. Iye, Phys. Rev. B 73, 052411 (2006).

${ }^{9}$ G. Möller and R. Moessner, Phys. Rev. Lett. 96, 237202 (2006).

${ }^{10}$ A. S. Wills, R. Ballou, and C. Lacroix, Phys. Rev. B 66, 144407
(2002).

${ }^{11}$ R. Milo, S. Shen-Orr, S. Itzkovitz, N. Kashtan, D. Chklovskii, and U. Alon, Science 298, 824 (2002).

${ }^{12}$ L. Grill, M. Dyer, L. Lafferentz, M. Persson, M. V. Peters, and S. Hecht, Nat. Nanotechnol. 2, 687 (2007).

${ }^{13}$ C. Quitmann, U. Flechsig, L. Patthey, T. Schmidt, G. Ingold, M. Howells, M. Janousch, and R. Abela, Surf. Sci. 480, 173 (2001).

${ }^{14}$ J. Stöhr, H. A. Padmore, S. Anders, T. Stammler, and M. R. Scheinfein, Surf. Rev. Lett. 5, 1297 (1998).

${ }^{15}$ R. P. Cowburn, D. K. Koltsov, A. O. Adeyeye, M. E. Welland, and D. M. Tricker, Phys. Rev. Lett. 83, 1042 (1999).

${ }^{16}$ J. Rothman, M. Kläui, L. Lopez-Diaz, C. A. F. Vaz, A. Bleloch, J. A. C. Bland, Z. Cui, and R. Speaks, Phys. Rev. Lett. 86, 1098 (2001).

${ }^{17}$ S. T. Bramwell, Nature (London) 439, 273 (2006).

${ }^{18}$ A. Imre, G. Csaba, L. Ji, A. Orlov, G. H. Bernstein, and W. Porod, Science 311, 205 (2006). 